\section{CROP GENETICS}

\section{Oil prospecting}

\section{Sci. Data 2, 150072 (2015)}

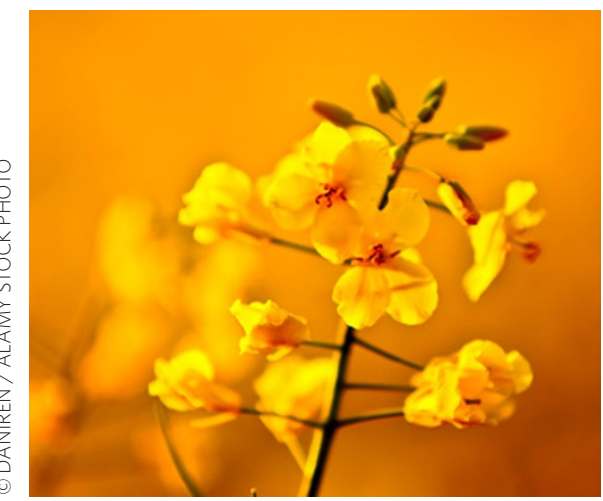

Crop improvement by selective breeding requires genetically diverse populations, but this selection tends to reduce such diversity. A detailed knowledge of the genome-level diversity available to breeders can help resolve this paradox. To this end, Thomas Schmutzer, Birgit Samans and colleagues have undertaken a comprehensive genome resequencing of a panel of 52 Brassica napus accessions and in the process identified 4.3 million highconfidence, genome-wide single nucleotide polymorphisms (SNPs).

Brassica napus - commonly known as oilseed rape or canola - is a major source of vegetable oil for food and biofuel. It is a tetraploid species created by the hybridization of B. rapa and B. oleracea around 7,500 years ago. To obtain the widest possible genetic range, Schmutzer et al. sequenced 32 crop varieties, including two elite modern lines and 20 'synthetic' B. napus accessions. These synthetic lines were created by de novo hybridization of B. rapa and B. oleracea, giving access to a greater diversity of the parental species than is present in cultivated $B$. napus.

Using next-generation sequencing, reads to an average depth of between $12 \mathrm{x}$ and $15 \mathrm{x}$ were obtained and aligned against a $B$. napus reference genome. These individual genome sequences and the associated SNPs will provide a foundation for screening and selection by crop breeders and a resource for exploring the genetics of polyploidy evolution.

\section{TOMATO DOMESTICATION}

\section{Slowing down the clock}

Nature Genet. http://doi.org/9z3 (2015)

Crop species undergo profound shifts in agronomic traits, such as architecture and fruit size, during domestication. Niels Müller of the Max Planck Institute for Plant Breeding Research, Cologne, and colleagues find that for tomatoes, the circadian clock also slowed down during domestication, probably allowing cultivation at higher latitudes.

The researchers observed that the circadian movements of leaves ran to a slower clock in cultivated tomatoes than in their wild ancestors. These had both a longer period and a delayed phase (time to maximum displacement). Similar deceleration was also seen in diurnal patterns of transcription.

\section{PHOTORESPIRATION}

\section{In the balance}

The balance between photosynthetic carbon gain and photorespiratory carbon loss is a key determinant of the productivity of $C_{3}$ plants. A study of plant stable isotope signatures suggests that the ratio of photorespiration to photosynthesis in $\mathrm{C}_{3}$ plants has fallen by around $25 \%$ since 1900 , in line with the 100 ppm rise in atmospheric carbon dioxide concentrations seen over the same period.

Ina Ehlers, of Umea University, Sweden, and colleagues carried out a series of laboratory experiments with sunflower to determine the intramolecular distribution of the stable isotope deuterium in photosynthetically derived glucose in plants exposed to different concentrations of carbon dioxide. They find that the intramolecular deuterium distribution shifts with $\mathrm{CO}_{2}$ concentration, and can thereby serve as a proxy for the ratio of photorespiration to photosynthesis. Applying this logic, they examine the deuterium signature of archived (field grown) sugar beet sugars collected between 1890 and 2012. They find that the ratio of photorespiration to photosynthesis declined significantly over the measurement period, such that net photosynthetic carbon gain rose by $35 \%$. Comparison of modern and herbarium samples of several other $C_{3}$ species revealed a similar trend.

The researchers suggest that their findings provide much-awaited experimental evidence for the ' $\mathrm{CO}_{2}$ fertilization' effect - the hypothesis that the post-industrial rise in atmospheric $\mathrm{CO}_{2}$ concentrations has stimulated plant carbon gain.
A gene, EID1, was identified by map-based cloning, whose 'wild' allele significantly advanced the phase when introduced into cultivated tomato. Moreover, a selective sweep was observed around EID1, indicating the cultivated allele is adaptive and has been selected for by humanity. Plants bearing the cultivated allele have higher chlorophyll content than those carrying the wild allele under long photoperiods, suggesting the slowed clock might prevent fitness loss under the long summer days encountered by domesticated tomatoes far from their equatorial origins.

Whether changes in the circadian clock are common during crop domestication is unknown; however, adaptation of a plant's daily rhythms may prove to be a fertile field of exploration.

\section{SIGNALLING}

\section{Poisoning the well \\ Plant Cell 27, 3175-3189 (2015)}

Allelopathy involves the production by plants such as grasses of secondary metabolites that disturb the growth or germination of their neighbours. This interaction often happens through root excretions that diffuse around the rhizosphere, and has ecological importance, as plants using it obtain a fitness advantage by reducing competition. It is crucial for structuring plant communities, including in agricultural ecosystems. Venturelli and colleagues have now discovered one molecular mechanism behind allelopathy that involves chromatin configuration.

Cyclic hydroxamic acids are one of the many substances released by roots. They are quickly degraded in the soil to stable chemical forms, which are potent inhibitors of the highly conserved chromatinmodifying histone deacetylases. In fact, these chemicals are as phytotoxic as a commercial herbicide. In Arabidopsis, they induce hyperacetylation for hundreds of genes, which then become over-activated, presumably perturbing plant growth and development.

Epigenetic modifications represent a wide-range layer of endogenous gene expression control. This makes dynamic chromatin remodelling an efficient target of choice for co-evolutionary adaptations by foreign organisms: pathogenic or symbiotic microbes, fungi, or in this case, other plants. Understanding this natural warfare can have practical applications, by increasing the repertoire of natural toxins used in agriculture and even in cancer research. 